\title{
Arterial Wall Dosimetry for Non-Hodgkin Lymphoma Patients Treated with Radioimmunotherapy
}

Robert F. Hobbs ${ }^{1}$, Sébastien Baechler ${ }^{2}$, Richard L. Wahl ${ }^{1}$, Bin He${ }^{1}$, Hong Song ${ }^{1}$, Caroline E. Esaias ${ }^{1}$, Eric C. Frey ${ }^{1}$, Heather Jacene ${ }^{1}$, and George Sgouros ${ }^{1}$

${ }^{1}$ Johns Hopkins University, Baltimore Maryland; and ${ }^{2}$ University Institute of Radiation Physics, University of Lausanne,

Lausanne, Switzerland

Tumors in non-Hodgkin lymphoma (NHL) patients are often proximal to the major blood vessels in the abdomen or neck. In external-beam radiotherapy, these tumors present a challenge because imaging resolution prevents the beam from being targeted to the tumor lesion without also irradiating the artery wall. This problem has led to potentially life-threatening delayed toxicity. Because radioimmunotherapy has resulted in long-term survival of NHL patients, we investigated whether the absorbed dose (AD) to the artery wall in radioimmunotherapy of $\mathrm{NHL}$ is of potential concern for delayed toxicity. SPECT resolution is not sufficient to enable dosimetric analysis of anatomic features of the thickness of the aortic wall. Therefore, we present a model of aortic wall toxicity based on data from 4 patients treated with ${ }^{131}$ I-tositumomab. Methods: Four NHL patients with periaortic tumors were administered pretherapeutic ${ }^{131}$ I-tositumomab. Abdominal SPECT and whole-body planar images were obtained at 48, 72, and $144 \mathrm{~h}$ after tracer administration. Blood-pool activity concentrations were obtained from regions of interest drawn on the heart on the planar images. Tumor and blood activity concentrations, scaled to therapeutic administered activities_-both standard and myeloablative-were input into a geometry and tracking model (GEANT, version 4) of the aorta. The simulated energy deposited in the arterial walls was collected and fitted, and the AD and biologic effective dose values to the aortic wall and tumors were obtained for standard therapeutic and hypothetical myeloablative administered activities. Results: Arterial wall ADs from standard therapy were lower (0.6-3.7 Gy) than those typical from external-beam therapy, as were the tumor ADs (1.4-10.5 Gy). The ratios of tumor AD to arterial wall $A D$ were greater for radioimmunotherapy by a factor of 1.9-4.0. For myeloablative therapy, artery wall ADs were in general less than those typical for external-beam therapy (9.4-11.4 Gy for 3 of 4 patients) but comparable for 1 patient (32.6 Gy). Conclusion: Blood vessel radiation dose can be estimated using the software package 3D-RD combined with GEANT modeling. The dosimetry analysis suggested that arterial wall toxicity is highly unlikely in standard dose radioimmunotherapy but should be considered a potential concern and limiting factor in myeloablative therapy.

Received Aug. 19, 2009; revision accepted Dec. 8, 2009.

For correspondence or reprints contact: George Sgouros, Department of Radiology, Johns Hopkins University, School of Medicine, CRB II 4M.61, 1550 Orleans St., Baltimore, MD 21231.

E-mail: gsgouro1@jhmi.edu

COPYRIGHT $\odot 2010$ by the Society of Nuclear Medicine, Inc.
Key Words: oncology; radiobiology/dosimetry; radionuclide therapy; SPECT/CT; Monte Carlo; lymphoma; radioimmunotherapy; toxicity

J Nucl Med 2010; 51:368-375

DOI: 10.2967/jnumed.109.069575

\section{D} uring the last $40 \mathrm{y}$, dramatic improvements have been achieved in the treatment of lymphomas (1), and although the life expectancy for a non-Hodgkin lymphoma (NHL) patient is less favorable than that for a patient with Hodgkin disease (HD), significant clinical progress and extended life expectancy is a reality today for both disease types. With improved survival, concerns and issues related to long-term toxicity and quality of life of the survivors have become crucial points of interest $(1,2)$.

Although external-beam therapy is used more in HD, both chemotherapy and radiation may cause short- and long-term side effects. In particular, arterial wall deterioration from external-beam radiation is well documented ( $1-$ 11); complications to the arteries can include arterial stenosis (3), atherosclerosis, stroke (4), aneurysm, and coronary heart disease $(5,6)$. Damage has been clinically reported to all the major arteries: coronary $(5,6)$, renal (7), aortic, femoral, and carotid (6). Studies have shown the time frame for toxicity responses to be as early as 1 y but more typically $10-20$ y or more after treatment $(1,6,8,9)$. Although initial postmortem studies evoked 35 Gy of standard fractionated external-beam radiation as a limit for arterial toxicity (10), increasing attention to long-term complications suggests lower thresholds (20-25 Gy) (6), more in line with values considered toxic $(\sim 27 \mathrm{~Gy})$ for other dose-limiting normal organs such as kidneys (12), liver, and lungs (13). Until recently, arterial toxicity typically has been considered only in cases of late complications and could be considered as a risk factor only in atherosclerosis and related vascular complications along with other treatment- and patient-related factors that affect 
the risk of late adverse outcomes, making estimation of toxicity thresholds difficult.

To overcome the problems of toxicity (threshold determination intrinsic to long-term clinical studies), animal studies have been conducted $(14,15)$. In a mouse model, a single exposure of 14 Gy was sufficient to induce significant arterial damage (14). Although direct translation of values from mouse to human is always questionable, this value merits attention.

Radioimmunotherapy, using anti-CD20 antibodies, has been approved as a treatment modality for NHL. ${ }^{131}$ I-labeled tositumomab (Bexxar; GlaxoSmithKline) has shown good therapeutic efficacy for patients with relapsed or refractory low-grade follicular NHL $(16,17)$, as has ${ }^{90}$ Y-ibrotumomab tiuxetan (Zevalin; Spectrum Pharmaceuticals, Inc.) $(18,19)$. With the increasing use of radioimmunotherapy as a viable treatment for NHL and the expected long-term survival of these patients, knowledge of the arterial wall absorbed dose (AD) in cases in which the disease sites are proximal to major blood vessels resulting from such treatment and the dose to the vessel from the blood is essential, a fortiori, for therapeutic regimens with high administered activities (AA). Myeloablative treatment, in which the administered activity is based on dose limitations to normal organs other than bone marrow $(20,21)$, is such a case.

To resolve this issue, recourse to standard dosimetric methodologies is insufficient, even those typically used in patient-specific 3-dimensional (3D) dosimetry. SPECT or PET resolution is not sufficient to enable standard dosimetric analysis of anatomic features of the thickness of the aortic wall, nor is any imaging modality with its cubic lattice and large voxels able to distinguish and define the artery walls. In this study, we have performed dosimetry on 4 patients with periaortic tumors treated with ${ }^{131}$ I-tositumomab and unlabeled tositumomab and calculated AD values to the aortic wall for both standard and myeloablative therapeutic AA by incorporating a Monte Carlo (MC)based geometry and tracking model (GEANT, version 4) into the 3D-RD dosimetry software $(22,23)$.

\section{MATERIALS AND METHODS}

\section{Patients}

Four patients of the 8 enrolled in an Institutional Review Board-approved NHL dose-response protocol presented with noticeable tumors in the abdominal cavity. In particular, during restaging with ${ }^{18} \mathrm{~F}-\mathrm{FDG}$ PET/CT images (Fig. 1) the tumor masses circumscribed a large portion of either the abdominal aorta or one or both femoral arteries. Patient clinical data pertinent to this study are given in Table 1 .

\section{Data Acquisition}

Each patient was administered approximately $200 \mathrm{MBq}$ of ${ }^{131} \mathrm{I}-$ tositumomab as a tracer. Abdominal SPECT/CT scans taken on a Precedence SPECT/CT (16-slice; Philips) camera and wholebody (WB) planar images acquired on a VG Millennium Hawkeye camera (GE Healthcare) were acquired at 1 (WB only), 48, 72, and $144 \mathrm{~h}$ after tracer administration.

All the SPECT images were reconstructed using the quantitative SPECT (24) method (30 iterations, 16 subsets per iteration), based on the iterative ordered-subsets expectation maximization algorithm (25) with reconstruction-based compensation for attenuation, scatter, and the collimator-detector response function. The attenuation was modeled using measured CT-based attenuation maps. Scatter compensation was performed using the effective source scatter estimation method with a fast implementation. Point sources at various distances from the face of the collimator were simulated to estimate the distance-dependent collimator-detector response function that included interactions and penetration of photons in the collimator and detector. Figure 2 illustrates examples of the SPECT reconstruction.

\section{Therapeutic Activity Calculation}

The standard therapeutic quantities of administered ${ }^{131} \mathrm{I}$ activity were determined from the WB tracer retention half-life calculated from WB images according to a protocol reported by Wahl (26), using the WB dose limits given in Table 1. The WB dose limits (65 or $75 \mathrm{cGy}$ ) were determined from the platelet counts of the patient shortly before administration of the pretherapeutic activity. The calculated therapeutic activities for the 4 patients are given in Table 1.

\section{D-RD}

The reconstructed images and the accompanying CT scans were registered on a HERMES workstation (HERMES Medical Solutions) by registering CT to $\mathrm{CT}$ and then loaded into the 3D-RD software $(22,23)$.

The 3 SPECT coregistered activity images and a coregistered CT scan were imported into the 3D-RD software package. Volumes of interest (VOIs) were drawn around the tumor and selected major organs (lungs, liver, kidneys, spleen) in each patient, based on the CT images. One million events were run for the $\beta^{-}$and photon components of the ${ }^{131}$ I decay spectra based on the SPECT images for each time point using electron $\gamma$-shower MC software. The energy deposition distribution from the
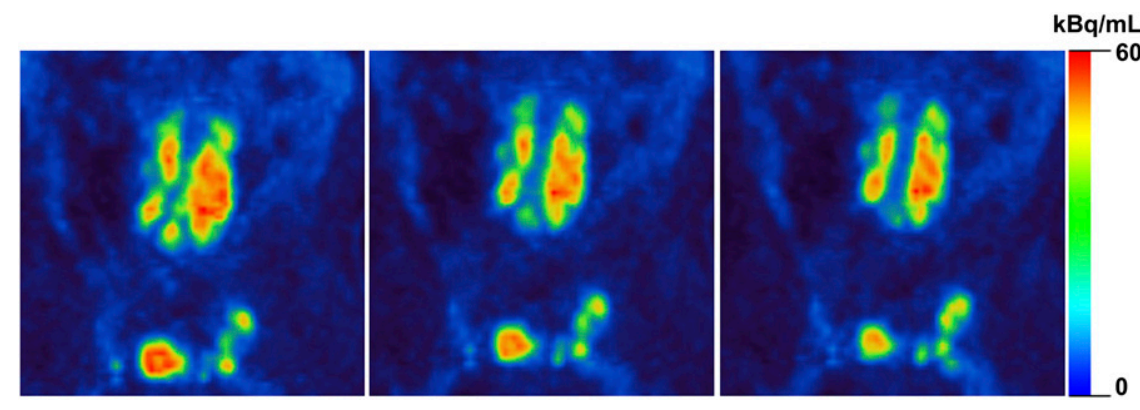

FIGURE 1. Consecutive coronal ${ }^{18} \mathrm{~F}-\mathrm{FDG}$ PET slices for restaging (patient 3). 


\begin{tabular}{|c|c|c|c|c|c|c|c|}
\hline Patient no. & Sex & Age $(y)$ & Height (cm) & Mass (kg) & Pretherapeutic AA (GBq) & WB limit (cGy) & Therapeutic AA (GBq) \\
\hline 1 & M & 68 & 170 & 90.5 & 0.192 & 65 & 3.03 \\
\hline 2 & $\mathrm{~F}$ & 63 & 147 & 64.5 & 0.204 & 65 & 2.28 \\
\hline 3 & M & 52 & 183 & 79 & 0.200 & 65 & 6.90 \\
\hline 4 & M & 63 & 178 & 78.5 & 0.196 & 75 & 3.72 \\
\hline
\end{tabular}

contributing components from MC were weighted for probability and therapeutic activity and converted to absorbed dose rate for the VOIs (spectra probability distributions obtained from the LBL/ Lund web site (27)). The dose rates for the different VOIs were fit with a hybrid trapezoid-exponential fit and integrated to obtain the AD.

\section{Hypothetical Myeloablative Activity Calculation}

To gauge the range of potential ADs to the artery walls from the most aggressive radioimmunotherapy treatment plan available, hypothetical therapeutic AAs used in myeloablative therapy were calculated using the guidelines established by the University of Washington (21). The administered therapeutic activity was scaled to deliver no more than the maximum tolerated dose (MTD), generally on the order of $27 \mathrm{~Gy}$, to the most vulnerable organ (excluding bone marrow), as determined by pretherapeutic dosimetry.

\section{Geometric Model}

The model for estimating the $\mathrm{AD}$ to the artery walls comprises 3 concentric cylinders created in GEANT, representing the arterial contents (blood), artery wall, and the tumor. Figure 3 illustrates the model and many of the parameters used. The parameters for the arteries are taken from the literature, and the parameters for the tumor are taken from the data. The volumes of the tumors, $\mathrm{V}$, were on the order of $300 \mathrm{~g}$ (261-424 g). A tumor thickness, $r_{t}$, of $3 \mathrm{~cm}$ was used; consequently, the height, $\mathrm{h}$, was approximately $10 \mathrm{~cm}$ for each tumor. The internal radius of the aorta, $\mathrm{R}$, was given a value of $6.93 \mathrm{~mm}$, and the thickness of the aorta wall, $\mathrm{r}_{\mathrm{w}}$, was $1.15 \mathrm{~mm}(28)$. The parameter $d$ in Figure 3 represents the range $(2 \mathrm{~cm})$ in the center of the model for which the aortic wall AD would be the greatest and for which the average aortic wall $\mathrm{AD}$ was calculated.

The second panel of Figure 3 represents a scenario with the same parameters for which the tumor circumscribes only half (50\%) of the vessel. In this scenario, the AD to the arterial wall was calculated only in the half adjacent to the tumor.

Additionally, another set of scenarios was studied, namely for tumors surrounding smaller arteries such as the femoral and carotid arteries. For these scenarios (for both $100 \%$ and $50 \%$ enclosures), $\mathrm{R}$ was changed to $3.26 \mathrm{~mm}, \mathrm{r}_{\mathrm{w}}$ became $0.91 \mathrm{~mm} \mathrm{(28),}$ and $r_{t}$ was also modified to $1.5 \mathrm{~cm}$, reflecting the smaller sizes of tumor seen in the PET images surrounding the smaller arteries.

\section{Kinetics}

${ }^{131}$ I activity concentration for the tumors for each time point was obtained from the SPECT images by dividing the measured activity by tumor volume, thus obtaining a uniform activity concentration for the model. Blood-pool activity concentrations were obtained from the WB images by drawing region-of-interest (ROI) contours around the heart, measuring the counts, subtracting background counts from a scaled background ROI, converting to activity by scaling to total AA divided by the total number of initial counts in the whole body, and dividing by a typical heart volume $(296 \mathrm{~mL})$. Tumor and blood activity concentrations were plotted as a function of time for each patient and integrated using either a simple exponential or a hybrid trapezoid-exponential fit. The area under the curves was taken and multiplied by the blood or tumor volume to obtain the respective cumulated activities for blood and tumor for each patient.

\section{$M C$}

GEANT MC simulations using 1 million decays were run separately for the tumor and blood (as defined in the model shown in Fig. 3) for each of the following scenario combinations: the aorta-sized vessel and tumor and the femoral artery-sized case, $50 \%$ and $100 \%$ enclosure scenarios, and ${ }^{131} \mathrm{I}$ and ${ }^{90} \mathrm{Y}$ cases.

The deposited energy was collected for each time point in the arterial walls (only in the central $2 \mathrm{~cm}$ for all cases and only in the half wall adjacent to the tumor for the $50 \%$ enclosure scenarios), the number of decays converted to units of cumulated activity, and the energy divided by mass to obtain the $\mathrm{S}$ values for the different cases. The ${ }^{90} \mathrm{Y}$ case was added, in consideration of the fact that the other radioimmunotherapeutic for $\mathrm{NHL}$ is ${ }^{90} \mathrm{Y}$-ibritumomab tiuxetan. Although we recognized that ${ }^{90}$ Y-ibritumomab tiuxetan uptake, kinetics, and AAs are different from those of ${ }^{131}$ I-tositumomab, rendering any direct dose comparison invalid,

FIGURE 2. Consecutive coronal slices of reconstructed SPECT images at $48 \mathrm{~h}$ for patient 3. CPS = counts per second.
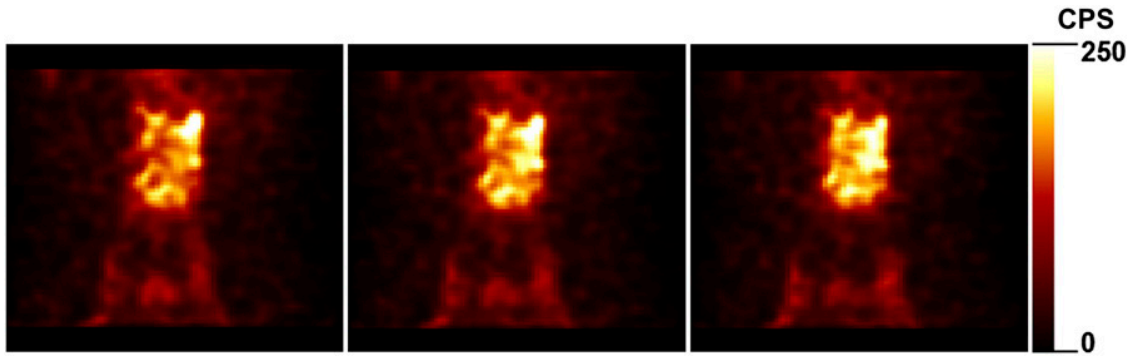


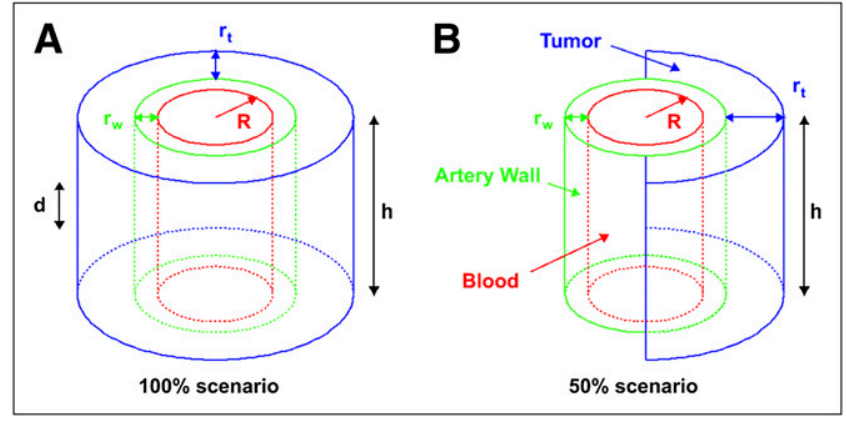

FIGURE 3. Geometric model of artery (red), arterial wall (green), and tumor (blue).

a comparison of the $\mathrm{S}$ values between the 2 isotopes could be considered instructive. Thus, ${ }^{90} \mathrm{Y}$ was included in the MC portion of the model but not in the subsequent dosimetric calculations.

AD

The ADs for the artery walls were obtained by multiplying the cumulated activities by the $\mathrm{S}$ values for the respective blood and tumor components and summing them. Additionally, a wholebody contribution was added to the $\mathrm{AD}$ to the arteries, the rationale being that the therapeutic AA is based on a limit on WB self-dose. This WB self-dose, which varies by patient as a function of platelet count, must be considered in the final AD calculation. However, a large fraction of the self-dose is from $\beta$ emissions (57\% as determined by calculating the photon component of the ${ }^{131}$ I whole-body-to-whole-body $\mathrm{S}$ value by subtracting from it the total electron energy emitted by ${ }^{131}$ I per decay divided by the whole-body mass), which are short-ranged and were considered much more specifically using the MC input values. The missing WB contribution was the longer-ranged photon component; consequently, $43 \%$ of the 65 or 75 cGy was added to the artery wall $\mathrm{AD}$.

\section{Radiobiology}

In addition to the $\mathrm{AD}, 3 \mathrm{D}-\mathrm{RD}$ incorporates radiobiologic modeling and calculates the biologic effective dose (BED) for all tumors and normal organs, including the modeled arterial wall. The BED $(29,30)$ relates dose and dose rate to the biologic effect it will have and has been shown to better predict normal-organ toxicity $(12,31)$. The formula for the BED is:

$$
\mathrm{BED}=\mathrm{D}\left(1+\frac{\mathrm{G}(\infty)}{\alpha / \beta} \mathrm{D}\right)
$$

where $\alpha$ and $\beta$ are the radiobiologic parameters from the linear quadratic model of cell survival (32), D is the absorbed dose, and $\mathrm{G}(\mathrm{T})$ is the Lea-Catcheside $\mathrm{G}$ factor:

$$
\mathrm{G}(\mathrm{T})=\frac{2}{\mathrm{D}^{2}} \int_{0}^{T} \dot{\mathrm{D}}(\mathrm{t}) \mathrm{dt} \int_{0}^{t} \dot{\mathrm{D}}(\mathrm{w}) \mathrm{e}^{-\mu(\mathrm{t}-\mathrm{w})} \mathrm{dw}, \quad \text { Eq. } 2
$$

where $\mu$ is the DNA repair constant, assuming exponential repair, and $t$ and $w$ are integration variables. The $\alpha$-to- $\beta$ ratio for NHL was $8.6 \mathrm{~Gy}$ (33), and for the artery wall, a value of 3.0 Gy was used (34). The repair rates typical for normal tissue (35) and tumor (36) used in this analysis were $0.46 \mathrm{~h}^{-1}$ and $1.3 \mathrm{~h}^{-1}$, respectively. The BED was calculated for the tumor in 3D-RD and for the aortic and arterial walls in the model using numeric integration (37). For external-beam radiation the formula becomes:

$$
\mathrm{BED}=\mathrm{D}\left(1+\frac{\mathrm{d}}{\alpha / \beta}\right)
$$

Eq. 3

where $\mathrm{d}=\mathrm{D} / \mathrm{N}$ is the dose delivered per session, typically 2 Gy. The ratio between BED to the tumor and BED to the artery wall is then:

$$
\mathrm{r}=\frac{\left(1+\frac{\mathrm{d}}{(\alpha / \beta)_{\text {tumor }}}\right)}{\left(1+\frac{\mathrm{d}}{(\alpha / \beta)_{\text {artery }}}\right)}
$$

\section{RESULTS}

\section{Model Kinetics}

The activity concentrations obtained for the blood from the WB planar images and for the tumors from the SPECT images are plotted and shown in Figure 4. The blood curves were fit with single exponential fits, and the tumor curves were fit with a single exponential for patients 2 and 3 only. Patient 1's tumor activity curve was fit with trapezoids to the last time point and then allowed to decay physically. Patient 4's curve was fit with trapezoids for the first 2 time

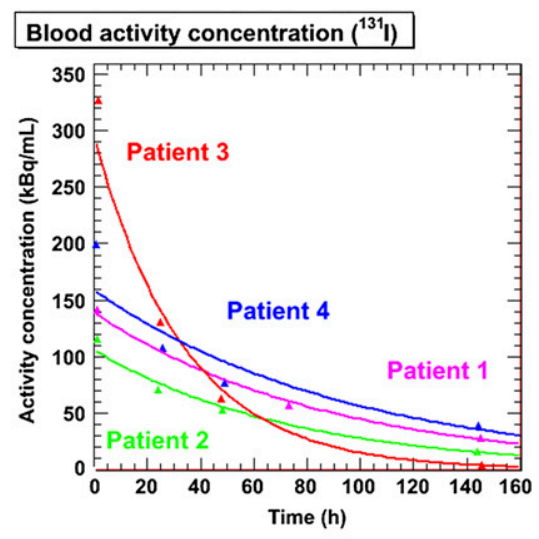

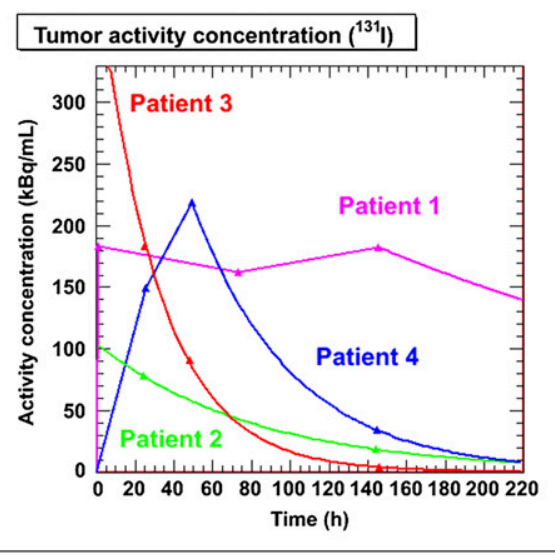

FIGURE 4. Model activity concentration kinetics in blood (left) and tumor (right) as determined from patient data (WB scans and SPECT images, respectively). 
TABLE 2. Patient-Fit Parameters for Blood and Tumor Kinetics Shown in Figure 4

\begin{tabular}{|c|c|c|c|c|c|c|}
\hline \multirow[b]{2}{*}{ Patient no. } & \multicolumn{3}{|c|}{ Blood } & \multicolumn{3}{|c|}{ Tumor } \\
\hline & $\overline{\mathrm{C}_{0}(\mathrm{kBq} / \mathrm{mL})}$ & $\lambda\left(h^{-1}\right)$ & $R^{2}$ & $\overline{\mathrm{C}_{0}(\mathrm{kBq} / \mathrm{mL})}$ & $\lambda\left(h^{-1}\right)$ & $\overline{R^{2}}$ \\
\hline 1 & 139 & $1.2410^{-2}$ & 0.99 & - & - & - \\
\hline 2 & 106 & $1.3110^{-2}$ & 0.99 & 104 & $1.2110^{-2}$ & 1 \\
\hline 3 & 295 & $2.7410^{-2}$ & 0.99 & 417 & $3.2510^{-2}$ & 0.99 \\
\hline 4 & 159 & $1.4810^{-2}$ & 0.91 & $219^{*}$ & $1.5410^{-2}$ & 1 \\
\hline
\end{tabular}

points and then fit to an exponential calculated between the last 2 time points. The parameters for the exponential fits are given in Table 2, where appropriate. The areas under the different curves were calculated and multiplied by the appropriate volumes to obtain cumulated activities.

$\mathrm{MC}$

The simulated energy depositions from the different MC scenarios were converted into $\mathrm{S}$ values, and the results are presented in Table 3.

\section{AD}

ADs to the artery walls were obtained as the product of the cumulated activities and the GEANT model-derived S values. Tumor, liver, lung, and kidney ADs were calculated using 3D-RD. The ADs to the latter 3 normal organs (values not shown) were used to determine the myeloblative AAs.

The ADs for the artery walls are given in Table 4; they are divided into dose contributions from whole-body photon emissions and from the total (electron and photon) contributions from the blood and tumor. The 3D-RD-derived tumor ADs for patients 1-4 were 10.5, 1.44, 2.12, and 3.14 Gy, respectively. The corresponding GEANT-modeled tumor ADs were 10.5, 1.44, 2.63, and 2.39 (simulated tumor ADs for other cases not shown).

Total ADs and BEDs are given in Table 5. The ratios between the $\mathrm{AD}$ to the (modeled) tumor and the $\mathrm{AD}$ to the artery wall are also given.

\section{BED}

To calculate the BED, not only the $\mathrm{AD}$ value but also the kinetics must be known. By multiplying the blood and tumor activities at the different time points by the appropriate $S$ values, the dose rate curves are obtained (Fig. 5). As expected, this figure resembles a linear combination of the 2 graphs in Figure 4. The BED is calculated from these curves by numeric integration; the results are presented in Table 5.

The conversion factor of AD to BED (Eq. 3) for external beam-assuming a single dose, d, of 2 Gy per patient visit—is 1.23 for the tumor and 1.67 for the artery wall. This represents a BED range of 33-67 Gy to the artery wall and 25-49 Gy to the tumor for a typical range of externalbeam treatment (20-40 Gy).

\section{Myeloablative BED}

For administration of myeloablative activities, the ADs to both the tumor and artery walls are simply multiplied by the multiplicative factor (therapeutic factor in Table 6). This factor was determined by scaling the AD values for the potential dose-limiting organs (kidneys, liver, or lungs) to the MTD and retaining the lowest scaling factor. The BEDs were recalculated numerically from the increased dose rate values. Table 6 shows the BED values calculated for myeloablative activities of ${ }^{131}$ I-tositumomab. For patient 1, the results in parentheses are those that consider the artery wall to be the dose-limiting organ: the AA has been scaled to deliver 27 Gy of BED to the artery wall.

\section{DISCUSSION}

\section{AD Comparison}

The calculated ADs from standard ${ }^{131}$ I-tositumomab radioimmunotherapy to the arterial walls were lower $(0.60-3.71 \mathrm{~Gy})$ than those typical from external-beam therapy (20-40 plus Gy) for the 4 patients examined, using GEANT MC modeling and 3D-RD dosimetry. Moreover, the typical artery AD is 1.9-4.0 times lower than the tumor dose in these worst-case scenarios (tumor immediately adjacent to artery) modeled here and is below the range

\begin{tabular}{|c|c|c|c|c|c|}
\hline Isotope & Case & Aorta $\leftarrow$ tumor & Aorta $\leftarrow$ blood & Femoral artery $\leftarrow$ tumor & Femoral artery $\leftarrow$ blood \\
\hline $131 \mid$ & $100 \%$ & 0.0986 & 0.964 & 0.280 & 4.22 \\
\hline 131 & $50 \%$ & 0.137 & 1.93 & 0.448 & 8.44 \\
\hline${ }^{90} Y$ & $100 \%$ & 0.528 & 11.4 & 2.44 & 45.6 \\
\hline${ }^{90} Y$ & $50 \%$ & 0.941 & 22.8 & 4.18 & 91.2 \\
\hline
\end{tabular}


TABLE 4. AD (Gy) from Blood, Tumor, and WB Photons to Aortic and Femoral Artery Wall

\begin{tabular}{|c|c|c|c|c|c|c|c|c|c|}
\hline \multirow[b]{2}{*}{ Patient no. } & \multirow[b]{2}{*}{$W^{*}$} & \multicolumn{2}{|c|}{ Aortic wall } & \multicolumn{2}{|c|}{ Half aortic wall } & \multicolumn{2}{|c|}{ Femoral wall } & \multicolumn{2}{|c|}{ Half femoral wall } \\
\hline & & Tumor & Blood & Tumor & Blood & Tumor & Blood & Tumor & Blood \\
\hline 1 & 0.28 & 3.25 & 0.18 & 2.25 & 0.18 & 2.33 & 0.17 & 1.87 & 0.17 \\
\hline 2 & 0.28 & 0.37 & 0.12 & 0.26 & 0.12 & 0.27 & 0.11 & 0.21 & 0.11 \\
\hline 3 & 0.28 & 0.75 & 0.14 & 0.52 & 0.14 & 0.54 & 0.14 & 0.43 & 0.14 \\
\hline 4 & 0.32 & 0.66 & 0.22 & 0.46 & 0.22 & 0.47 & 0.22 & 0.38 & 0.22 \\
\hline Average & 0.29 & 1.26 & 0.17 & 0.87 & 0.17 & 0.90 & 0.16 & 0.72 & 0.16 \\
\hline
\end{tabular}

of values considered as potentially toxic for fractionated external-beam therapy (20-35 Gy). For tumor masses that are near although not immediately adjacent to the vessel wall, the ratio of tumor dose to artery wall dose increases dramatically, resulting in even less potential long-term toxicity. This is in contrast to external-beam therapy, for which improvements in radiation technology such as intensity-modulated radiation therapy, designed to reduce the dose to regions outside the targeted area, often fail to reduce the dose to proximal organs (38) — even those that can be drawn and defined on the treatment planning, which certainly excludes artery walls. The ratio of tumor dose to artery wall dose in those cases is effectively 1.0.

\section{Myeloablative Therapy}

The use of myeloablative therapy has proven to be successful $(39,40)$, with limited toxicities (20), although studies examining long-term (10-20 y) side effects have yet to be performed. For 3 of 4 hypothetical treatments, arterial wall BED was below the standard 27 Gy of normal-organ toxicity (9.7-12.5 Gy). For patient 1, including the artery wall in the list of potential dose-limiting organs would have limited the AA to $20.5 \mathrm{GBq}$ instead of $26.7 \mathrm{GBq}$, assuming a limiting BED of $27 \mathrm{~Gy}$, suggesting the utility of such an inclusion. The range of hypothetical AAs calculated in the present study was higher (mean, $40.0 \mathrm{GBq}$; range, 26.7$70.6 \mathrm{GBq}$ ) than for the published results (mean, $20.3 \mathrm{GBq}$; range, 9.6-42.7 GBq) (21). Those results, however, used a range of MTD dose values (21-27 Gy) rather than a fixed $27 \mathrm{~Gy}$, and the mean $\mathrm{AA}$ of the present study was somewhat skewed by a single outlier (70.6 GBq), with a value unlikely to be administered in a real clinical setting.

\section{BED Comparison}

Comparison with BED values is important in accounting for the differences in dose rates: the BED to the arterial walls is 1.35 times greater than the BED to the tumor (ratio of 0.74 ) and 1.67 times more than the $\mathrm{AD}$ to the artery walls for external-beam therapy. This result is dependent on the accuracy of the $\alpha / \beta$ values, which are determined for cell lines in vitro and carry a significant degree of uncertainty; if the $\alpha / \beta$ value were lower for the tumor, this would bring the BED ratio between tumor and artery wall closer to 1. Additionally, the BED values to tumor and artery wall increase if the dose per fraction, $d$, in externalbeam radiotherapy increases. The ratio between $\mathrm{AD}$ and BED to the artery wall for $\mathrm{d}=3 \mathrm{~Gy}$ increases from 1.67 to 2.0 , resulting in a potential BED of $80 \mathrm{~Gy}$ to the artery wall for an $\mathrm{AD}$ of $40 \mathrm{~Gy}$ to a tumor proximal to the artery. In contrast, the BEDs to the artery walls for the 4 patients in this study are $0.60-3.75 \mathrm{~Gy}$. The ratios of tumor to artery wall BED are on the same order as the tumor-to-artery wall $\mathrm{AD}$ ratios.

\section{$S$ Values}

The $\mathrm{S}$ value comparison between ${ }^{90} \mathrm{Y}$ and ${ }^{131} \mathrm{I}$ reveals greater values for ${ }^{90} \mathrm{Y}$, about an order of magnitude, particularly the values from the blood. This must be taken in context with the relatively lower AA (approximately 3 times less) typical for ${ }^{90}$ Y-ibrotumomab tiuxetan therapy with respect to ${ }^{131} \mathrm{I}$-tositumomab. Although the blood $\mathrm{S}$ values are fixed, the relative weight of the tumor $S$ values is

TABLE 5. Modeled Arterial Wall ADs, BEDs, and Ratios of Tumor $A D$ to Arterial Wall $A D\left(D_{t} / D_{a}\right)$ Calculated from GEANT

\begin{tabular}{|c|c|c|c|c|c|c|c|c|c|c|c|c|}
\hline \multirow[b]{3}{*}{ Patient no. } & \multicolumn{6}{|c|}{ Aortic wall } & \multicolumn{6}{|c|}{ Femoral wall } \\
\hline & \multicolumn{3}{|c|}{$100 \%$} & \multicolumn{3}{|c|}{$50 \%$} & \multicolumn{3}{|c|}{$100 \%$} & \multicolumn{3}{|c|}{$50 \%$} \\
\hline & AD (Gy) & BED (Gy) & $D_{t} / D_{a}$ & $\overline{A D}(G y)$ & BED (Gy) & $\overline{D_{t} / D_{a}}$ & $\overline{A D}(G y)$ & BED (Gy) & $\overline{D_{t} / D_{a}}$ & AD (Gy) & BED (Gy) & $D_{t} / D_{a}$ \\
\hline 1 & 3.71 & 3.75 & 2.83 & 2.71 & 2.73 & 3.69 & 2.78 & 2.81 & 3.47 & 2.32 & 2.33 & 4.00 \\
\hline 2 & 0.77 & 0.77 & 1.88 & 0.65 & 0.65 & 2.13 & 0.66 & 0.66 & 2.05 & 0.60 & 0.60 & 2.16 \\
\hline 3 & 1.17 & 1.19 & 2.24 & 0.94 & 0.95 & 2.67 & 0.96 & 0.97 & 2.55 & 0.85 & 0.86 & 2.77 \\
\hline 4 & 1.20 & 1.21 & 1.98 & 1.00 & 1.00 & 2.29 & 1.01 & 1.02 & 2.20 & 0.92 & 0.93 & 2.34 \\
\hline Average & 1.71 & 1.73 & 2.23 & 1.33 & 1.33 & 2.70 & 1.35 & 1.37 & 2.57 & 1.17 & 1.18 & 2.82 \\
\hline
\end{tabular}




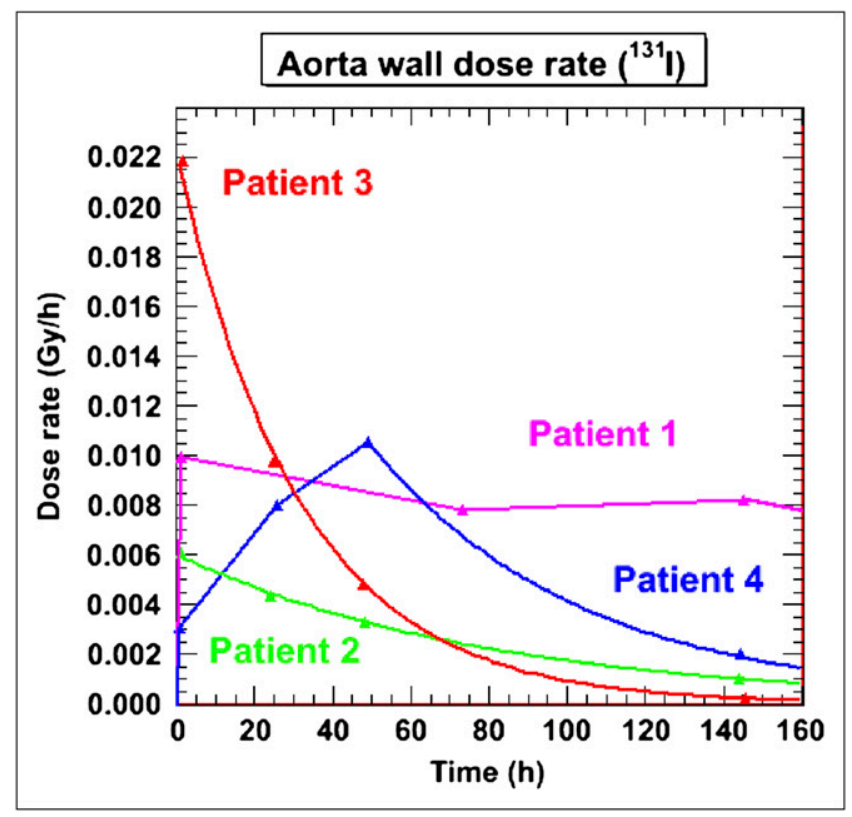

FIGURE 5. Dose-rate plot for aortic model with $100 \%$ tumor enclosure and ${ }^{131}$ I-tositumomab.

likely to change as the distance between tumor and artery wall increases, even for small distances $(<1 \mathrm{~cm})$.

The $50 \%$ cases have approximately twice the $\mathrm{S}$ value of the $100 \%$ cases because the artery wall mass used in the calculations is only that adjacent to the tumor-that is, half that taken for the $100 \%$ case. The relative significance of damage to a range of percentages of the circumference of the artery wall is unclear yet obviously of great importance to the clinical impact.

The source volumes for the blood-to-wall $\mathrm{S}$ values are those described in Figure 3 and not the whole blood volume. The blood-to-artery wall $\mathrm{S}$ values are significantly larger than the tumor-to-artery wall S values (Table 5). The $\mathrm{AD}$ results (Table 6), however, indicate a much greater contribution from the tumor. This greater contribution is due to the much larger volume of the tumor and, consequently, larger cumulated activity.

\section{Scope and Limitations}

The diameter used for the femoral artery is on a par with the diameters of the major arteries under discussion in this study: carotid, renal, and coronary, and thus the AD values can be considered reasonably relevant for all of these cases. The BED values depend on the accuracy of the $\alpha / \beta$ values found in the literature. Clearly, a different value from the one used here could influence the values calculated for the BED. A much higher value would dramatically reduce the ratio between $\mathrm{AD}$ and $\mathrm{BED}$ for external-beam therapy and reduce the BED from radioimmunotherapy slightly as well.

MC

This work illustrates a hybrid approach to AD calculations in situations where imaging cannot resolve the potential dose-limiting structure. The use of MC with idealized geometries overcomes the limitations of imaging-based dosimetry due to the finite resolving power of SPECT or PET.

\section{CONCLUSION}

Long-term toxicity from radiation to the artery walls for patients treated for NHL is not a concern with radioimmunotherapy, unlike for external-beam therapy. For myeloablative administered activities, the ADs to the artery walls from periarterial tumors may be commensurate with the lower end of toxic values; consequently, the artery wall should be considered a potential dose-limiting organ.

Patient-specific, 3D-RD dosimetry, combined with MC modeling, can provide important and more accurate dosimetry for anatomic features smaller than the imaging resolution.

\section{ACKNOWLEDGMENTS}

We thank Andrew Prideaux, Yuan-ping (Peter) Li, and Angelo Sibilio for their assistance. This study was funded in part by NIH NCI grant R01CA116477 and GlaxoSmithKline. Dr. Richard Wahl holds patents on both ${ }^{131}$ I-tositumomab and ${ }^{90}$ Y-ibritumomab tiuxetan and receives royalties via a licensing agreement when ${ }^{131} \mathrm{I}-$ tositumomab and ${ }^{90}$ Y-ibritumomab tiuxetan are used clinically in the United States. He also has received speaker fees from GlaxoSmithKline.

TABLE 6. Modeled Arterial Wall AD, BED, and Ratios of Tumor BED to Arterial Wall BED (BED $\left./ B^{\prime} D_{a}\right)$ Calculated from GEANT for Myeloablative AAs

$\begin{array}{lcccccc}\text { Patient } & \text { AA (GBq) } & \text { Therapeutic factor } & \text { Tumor BED (Gy) } & \text { Wall AD (Gy) } & \text { Wall BED (Gy) } & \text { BED } / \text { BED }_{a}(\mathrm{~Gy}) \\ 1 & 26.7(20.5) & 8.80(6.75) & 92.4(70.5) & 32.6(25.6) & 34.8(27.0) & 2.66(2.68) \\ 2 & 27.9 & 12.3 & 17.9 & 9.38 & 9.70 & 1.85 \\ 3 & 70.6 & 10.2 & 23.3 & 11.4 & 12.5 & 1.86 \\ 4 & 34.8 & 9.37 & 30.8 & 11.3 & 11.7 & 2.63 \\ \text { Average } & 40.0 & 10.2 & 41.1 & 16.2 & 17.2 & 2.25\end{array}$

Values in parentheses for patient 1 correspond to those obtained for treatment plan based on 27-Gy maximum BED to arterial wall. 


\section{REFERENCES}

1. von der Weid NX. Adult life after surviving lymphoma in childhood. Support Care Cancer. 2008;16:339-345.

2. Bowers DC, McNeil DE, Liu Y, et al. Stroke as a late treatment effect of Hodgkin's disease: a report from the Childhood Cancer Survivor Study. J Clin Oncol. 2005;23:6508-6515.

3. Rademaker J, Schoder H, Ariaratnam NS, et al. Coronary artery disease after radiation therapy for Hodgkin's lymphoma: coronary CT angiography findings and calcium scores in nine asymptomatic patients. AJR. 2008;191:32-37.

4. Scott AS, Parr LA, Johnstone PA. Risk of cerebrovascular events after neck and supraclavicular radiotherapy: a systematic review. Radiother Oncol. 2009;90: $163-165$.

5. Heidenreich PA, Schnittger I, Strauss HW, et al. Screening for coronary artery disease after mediastinal irradiation for Hodgkin's disease. J Clin Oncol. 2007;25:43-49.

6. Hull MC, Morris CG, Pepine CJ, Mendenhall NP. Valvular dysfunction and carotid, subclavian, and coronary artery disease in survivors of Hodgkin lymphoma treated with radiation therapy. JAMA. 2003;290:2831-2837.

7. Izzedine H, Cluzel P, Deray G. Renal radiation-induced arterial stenosis. Kidney Int. 2007;71:1188

8. Andersson A, Naslund U, Tavelin B, Enblad G, Gustavsson A, Malmer B. Longterm risk of cardiovascular disease in Hodgkin lymphoma survivors: retrospective cohort analyses and a concept for prospective intervention. Int J Cancer. 2009;124:1914-1917.

9. Patel DA, Kochanski J, Suen AW, Fajardo LF, Hancock SL, Knox SJ. Clinical manifestations of noncoronary atherosclerotic vascular disease after moderate dose irradiation. Cancer. 2006;106:718-725.

10. Brosius FC III, Waller BF, Roberts WC. Radiation heart disease: analysis of 16 young (aged 15 to 33 years) necropsy patients who received over 3,500 rads to the heart. Am J Med. 1981;70:519-530.

11. Chow LM, Nathan PC, Hodgson DC, et al. Survival and late effects in children with Hodgkin's lymphoma treated with MOPP/ABV and low-dose, extendedfield irradiation. J Clin Oncol. 2006;24:5735-5741.

12. Barone R, Borson-Chazot F, Valkema R, et al. Patient-specific dosimetry in predicting renal toxicity with ${ }^{90}$ Y-DOTATOC: relevance of kidney volume and dose rate in finding a dose-effect relationship. J Nucl Med. 2005;46(suppl 1):99S-106S.

13. Press OW, Eary JF, Appelbaum FR, et al. Radiolabeled-antibody therapy of Bcell lymphoma with autologous bone marrow support. N Engl J Med. 1993; 329:1219-1224.

14. Hoving S, Heeneman S, Gijbels MJ, et al. Single-dose and fractionated irradiation promote initiation and progression of atherosclerosis and induce an inflammatory plaque phenotype in ApoE(-/-) mice. Int J Radiat Oncol Biol Phys. 2008;71:848-857.

15. Pakala R, Leborgne L, Cheneau E, et al. Radiation-induced atherosclerotic plaque progression in a hypercholesterolemic rabbit: a prospective vulnerable plaque model? Cardiovasc Radiat Med. 2003;4:146-151.

16. Kaminski MS, Tuck M, Estes J, et al. ${ }^{131} \mathrm{I}$-tositumomab therapy as initial treatment for follicular lymphoma. N Engl J Med. 2005;352:441-449.

17. Vose JM, Wahl RL, Saleh M, et al. Multicenter phase II study of iodine-131 tositumomab for chemotherapy-relapsed/refractory low-grade and transformed low-grade B-cell non-Hodgkin's lymphomas. J Clin Oncol. 2000;18:1316-1323.

18. Wiseman GA, Kornmehl E, Leigh B, et al. Radiation dosimetry results and safety correlations from ${ }^{90} \mathrm{Y}$-ibritumomab tiuxetan radioimmunotherapy for relapsed or refractory non-Hodgkin's lymphoma: combined data from 4 clinical trials. J Nucl Med. 2003;44:465-474.

19. Witzig TE, Gordon LI, Cabanillas F, et al. Randomized controlled trial of yttrium-90-labeled ibritumomab tiuxetan radioimmunotherapy versus rituximab immunotherapy for patients with relapsed or refractory low-grade, follicular, or transformed B-cell non-Hodgkin's lymphoma. J Clin Oncol. 2002;20:2453-2463.
20. Liu SY, Eary JF, Petersdorf SH, et al. Follow-up of relapsed B-cell lymphoma patients treated with iodine-131-labeled anti-CD20 antibody and autologous stem-cell rescue. J Clin Oncol. 1998;16:3270-3278.

21. Rajendran JG, Gopal AK, Fisher DR, Durack LD, Gooley TA, Press OW. Myeloablative ${ }^{131}$ I-tositumomab radioimmunotherapy in treating non-Hodgkin's lymphoma: comparison of dosimetry based on whole-body retention and dose to critical organ receiving the highest dose. J Nucl Med. 2008;49:837844.

22. Prideaux AR, Song H, Hobbs RF, et al. Three-dimensional radiobiologic dosimetry: application of radiobiologic modeling to patient-specific 3dimensional imaging-based internal dosimetry. J Nucl Med. 2007;48:10081016.

23. Hobbs RF, Wahl RL, Lodge MA, et al. ${ }^{124}$ I PET-based 3D-RD dosimetry for a pediatric thyroid cancer patient: real-time treatment planning and methodologic comparison. J Nucl Med. 2009;50:1844-1847.

24. He B, Du Y, Song XY, Segars WP, Frey EC. A Monte Carlo and physical phantom evaluation of quantitative In-111 SPECT. Phys Med Biol. 2005;50: 4169-4185.

25. Hudson HM, Larkin RS. Accelerated image-reconstruction using ordered subsets of projection data. IEEE Trans Med Imaging. 1994;13:601-609.

26. Wahl RL. Tositumomab and ${ }^{131}$ I therapy in non-Hodgkin's lymphoma. $J$ Nucl Med. 2005;46(suppl 1):128S-140S.

27. Ekström L, Firestone R. WWW Table of Radioactive Isotopes: Nuclide Search. Available at: http://ie.lbl.gov/toi/nucSearch.asp. Accessed February 1, 2010.

28. International Commission on Radiological Protection. Reference Man: Anatomical, Physiological, and Metabolic Characteristics. ICRP publication 23. Oxford, U.K.: Elsevier; 1975.

29. Dale R. Use of the linear-quadratic radiobiological model for quantifying kidney response in targeted radiotherapy. Cancer Biother Radiopharm. 2004;19:363370 .

30. Baechler S, Hobbs RF, Prideaux AR, Wahl RL, Sgouros G. Extension of the biological effective dose to the MIRD schema and possible implications in radionuclide therapy dosimetry. Med Phys. 2008;35:1123-1134.

31. Wessels BW, Konijnenberg MW, Dale RG, et al. MIRD pamphlet no. 20: the effect of model assumptions on kidney dosimetry and response-implications for radionuclide therapy. J Nucl Med. 2008;49:1884-1899.

32. Fowler JF. The linear-quadratic formula and progress in fractionated radiotherapy. Br J Radiol. 1989;62:679-694.

33. Aref A, Mohammad R, Yudelev M, et al. Radiobiological characterization of two human chemotherapy-resistant intermediate grade non-Hodgkin's lymphoma cell lines. Radiat Oncol Investig. 1999;7:158-162.

34. Brenner DJ, Miller RC, Hall EJ. The radiobiology of intravascular irradiation. Int J Radiat Oncol Biol Phys. 1996;36:805-810.

35. Brenner DJ, Hall EJ. Conditions for the equivalence of continuous to pulsed low dose rate brachytherapy. Int J Radiat Oncol Biol Phys. 1991;20:181-190.

36. Bodey RK, Flux GD, Evans PM. Combining dosimetry for targeted radionuclide and external beam therapies using the biologically effective dose. Cancer Biother Radiopharm. 2003;18:89-97.

37. Hobbs RF, Sgouros G. Calculation of the biological effective dose (BED) for piece-wise defined dose-rate fits. Med Phys. 2009;36:904-907.

38. Goyal S, Cohler A, Camporeale J, Narra V, Yue NJ. Intensity-modulated radiation therapy for orbital lymphoma. Radiat Med. 2008;26:573-581.

39. Gopal AK, Gooley TA, Maloney DG, et al. High-dose radioimmunotherapy versus conventional high-dose therapy and autologous hematopoietic stem cell transplantation for relapsed follicular non-Hodgkin lymphoma: a multivariable cohort analysis. Blood. 2003;102:2351-2357.

40. Gopal AK, Rajendran JG, Gooley TA, et al. High-dose $\left[{ }^{131} \mathrm{I}\right]$ tositumomab (anti$\mathrm{CD} 20$ ) radioimmunotherapy and autologous hematopoietic stem-cell transplantation for adults $>$ or $=60$ years old with relapsed or refractory B-cell lymphoma. J Clin Oncol. 2007;25:1396-1402. 